\title{
QUALITATIVE ASSESSMENT OF INTRINSIC MODE FUNCTIONS OF EMPIRICAL MODE DECOMPOSITION
}

\author{
Mo Chen, Danilo P. Mandic \\ Imperial College London \\ Dept. of Electrical Engineering, \\ Exhibition Road, London, SW7 2BT, U.K. \\ E-mail: \{mo.chen, d.mandic\}@imperial.ac.uk
}

\author{
Preben Kidmose and Michael Ungstrup \\ Widex A/S, \\ Ny Vestergaardsvej 25, \\ 3500 Vaerloese, Denmark \\ E-mail: \{p.kidmose, miu\}@widex.dk
}

\begin{abstract}
The 'empirical mode decomposition' (EMD) method has been recently proposed to deal with nonlinear and nonstationary data, which decomposes signals into 'well-behaved' intrinsic mode functions (IMFs). An assessment on the qualitative performance of the EMD method in terms of the degree of signal nature preservation of individual IMF is provided. This is archived by means of the recently proposed signal characterisation method, based upon examining the signal predicability in phase space. It is shown that the first IMF always performs best in terms of signal nature preserving. Simulation results on both linear and nonlinear benchmark signals support the analysis.
\end{abstract}

Index Terms - Empirical mode decomposition (EMD), delay vector variance (DVV), intrinsic mode function (IMF), nonlinear, non-stationary

\section{INTRODUCTION}

Traditionally Fourier spectral analysis has been the dominant method used in the data analysis of the majority of signals; however it has its limitations and is only valid under very general conditions requiring a linear system and strictly periodic or stationary data. In more recent times, the emergence of wavelet analysis, the Wagner-Ville distribution and other time frequency methods [1][2] provide an appealing feature of being able to analyse non-stationary but linear data [3].

Although well accepted and extremely popular, both of the above a priori basis based data expansion techniques when applied to real world non-stationary and nonlinear signals results in data that may be insufficient and meaningless or even misleading. Furthermore, such methods suffer from difficulties of being non-adaptive in nature. An a posteriori basis (that is derived from the data and is therefore data-dependent) facilitates an adaptive data analysis method. The combination of the Empirical Mode Decomposition and Hilbert spectral analysis however is able to meet these requirements.

On the other hand, real-world processes comprise both linear and nonlinear components, together with deterministic and stochastic ones, yet it is a common practice to model such processes using suboptimal, but mathematically tractable models. Indeed, in the absence of nonlinear behaviour, it is not favourable to use nonlinear models, since these are more difficult to train than their linear counterparts, due to issues such as overfitting and computational complexity. Notice also that e.g. in biomedical applications such as in the analysis of the electrocardiogram (ECG) and electro-encephalogram (EEG), the linear/nonlinear nature of the signal conveys information concerning the health condition of a subject [4].

Although, the recently proposed EMD method is shown to be able to process data without any assumption on the linearity or stationarity. However, it is largely unknown whether IMFs after the decomposition preserve the nature ${ }^{1}$ of the original signal. To this end, we set out to investigate how different the individual IMF behaves in signal nature preserving.

\section{THE EMPIRICAL MODE DECOMPOSITION (EMD) METHOD}

EMD [3] is a method of breaking down a signal without leaving the time domain. It can be compared to other analysis methods like Fourier Transforms and wavelet decomposition. The process is useful for analysing natural signals, which are most often non-linear and non-stationary. Table 1 compares the EMD and Hilbert spectral analysis with the Fourier transform and wavelet method as discussed by Huang et al. [6].

EMD filters out functions which form a complete and nearly orthogonal basis for the original signal. Completeness is based on the method of the EMD; the way it is decomposed implies completeness. The functions, known as 'intrinsic mode functions' (IMFs), are therefore sufficient to describe the signal, even though they are not necessarily orthogonal. The real meaning here applies only locally. For some special data, the neighbouring components could certainly have sections of data carrying the same frequency at different time durations. But locally, any two components should be

\footnotetext{
${ }^{1}$ By the signal 'nature', we adhere to a number of signal properties described in [5]: linear, nonlinear, deterministic and stochastic signal behaviour.
} 


\begin{tabular}{|l|l|l|l|}
\hline Basis & Fourier & Wavelet & Hilbert \\
\hline $\begin{array}{l}\text { Frequency } \\
\text { scale }\end{array}$ & $\begin{array}{l}\text { A priori } \\
\text { Global } \\
\text { Uncertainty }\end{array}$ & $\begin{array}{l}\text { A priori } \\
\text { (adaptive) }\end{array}$ \\
\hline $\begin{array}{l}\text { Spectral } \\
\text { Uncertainty }\end{array}$ & $\begin{array}{l}\text { Energy- } \\
\text { frequency }\end{array}$ & $\begin{array}{l}\text { Energy-time- } \\
\text { frequency }\end{array}$ & $\begin{array}{l}\text { Energal } \\
\text { Certainty } \\
\text { frequency }\end{array}$ \\
\hline $\begin{array}{l}\text { Nonlinear } \\
\text { model }\end{array}$ & No & No & Yes \\
\hline $\begin{array}{l}\text { Nonstationary } \\
\text { model }\end{array}$ & No & Yes & Yes \\
\hline $\begin{array}{l}\text { Theoretical } \\
\text { base }\end{array}$ & Complete & Complete & Empirical \\
\hline
\end{tabular}

The sifting process continues until any of the following predetermined criteria is met: either when the component $c_{n}$ becomes so small than the predetermined value of substantial consequence, or when the residue, $r_{n}$, becomes a monotonic function from which no more IMF can be extracted. Even for data with zero mean the final residue can still be different from zero; for data with a trend, then the final residue should be that trend.

\section{THE "DELAY VECTOR VARIANCE" (DVV) METHOD}

We now describe the signal characterisation tool, 'delay vector variance' (DVV) method [5], based upon examining the predictability of a signal in the phase space, and examines simultaneously the determinism and nonlinearity within a signal. This method can be summarised as follows: For an opti$\mathrm{mal}^{2}$ embedding dimension $m$ :

- The mean, $\mu_{\mathrm{d}}$, and standard deviation, $\sigma_{\mathrm{d}}$, are computed over all pairwise Euclidean distances between delay vectors (DVs), $\|\mathbf{x}(i)-\mathbf{x}(j)\|(i \neq j)$.

- The sets $\Omega_{k}\left(r_{\mathrm{d}}\right)$ are generated such that $\Omega_{k}\left(r_{\mathrm{d}}\right)=$ $\left\{\mathbf{x}(i) \mid\|\mathbf{x}(k)-\mathbf{x}(i)\| \leq r_{\mathrm{d}}\right\}$, i.e., sets which consist of all DVs that lie closer to $\mathbf{x}(k)$ than a certain distance $r_{\mathrm{d}}$, taken from the interval $\left[\max \left\{0, \mu_{\mathrm{d}}-n_{\mathrm{d}} \sigma_{\mathrm{d}}\right\} ; \mu_{\mathrm{d}}+\right.$ $\left.n_{\mathrm{d}} \sigma_{\mathrm{d}}\right]$, e.g., $N_{\mathrm{tv}}$ uniformly spaced distances, where $n_{\mathrm{d}}$ is a parameter controlling the span over which to perform the DVV analysis.

- For every set $\Omega_{k}\left(r_{\mathrm{d}}\right)$, the variance of the corresponding targets, $\sigma_{k}^{2}\left(r_{\mathrm{d}}\right)$, is computed. The average over all sets $\Omega_{k}\left(r_{\mathrm{d}}\right)$, normalised by the variance of the time series, $\sigma_{x}^{2}$, yields the 'target variance', $\sigma^{* 2}\left(r_{\mathrm{d}}\right)$ :

$$
\sigma^{* 2}\left(r_{\mathrm{d}}\right)=\frac{\frac{1}{N} \sum_{k=1}^{N} \sigma_{k}^{2}\left(r_{\mathrm{d}}\right)}{\sigma_{x}^{2}} .
$$

To illustrate the meaning of 'signal nature' and the usage of DVV method, consider a linear benchmark signal (AR(4)) [8], given by

$$
\begin{aligned}
x(k) & =1.79 x(k-1)-1.85 x(k-2)+1.27 x(k-3) \\
& -0.41 x(k-4)+n(k)
\end{aligned}
$$

where $n(k) \sim \mathcal{N}(0,1)$ and a chaotic nonlinear signal, $\mathrm{x}$ component of Henon map [9], given by

$$
\begin{aligned}
& x_{n}=1-a x_{n-\tau}^{2}+y_{n-\tau} \\
& y_{n}=b x_{n-\tau}
\end{aligned}
$$

where $\tau$ is the time lag which was set to unity, and parameters $a$ and $b$ were set to 1.4 and 0.3 , respectively. We will refer to this signal as the Henon map though only the $\mathrm{x}$-component of Henon map is used in the remainder of the paper.

\footnotetext{
${ }^{2}$ We adopt Cao's method [7] for choosing the optimal embedding dimension in all of our simulations, which yields four for the linear benchmark signal (2) and two for the nonlinear benchmark signal (3).
} 


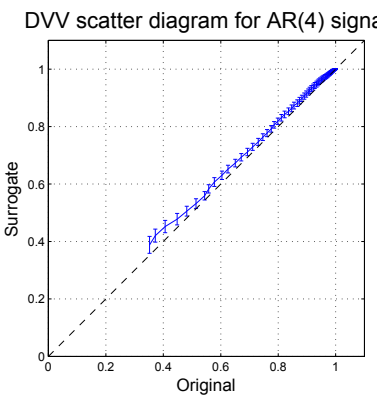

(a)

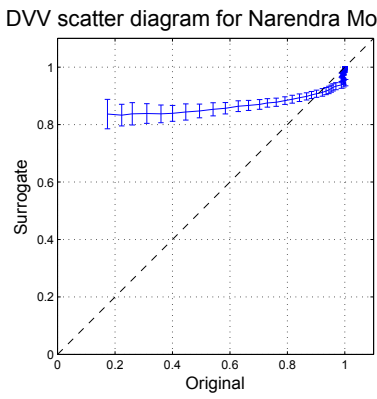

(b)
Fig. 1. DVV scatter diagrams for AR(4) signal (left diagram) and the Henon map signal (right diagram).

In the following step, DVV scatter diagram is constructed in the way where the horizontal axis corresponds to the target variance $\left(\sigma^{* 2}\left(r_{\mathrm{d}}\right)\right)$ of the original time series, and the vertical to that of the surrogate time series. A linear signal will have similar target variance as its surrogate, resulting in the DVV scatter diagram coinciding with the bisector line as illustrated in Figure 1(a), whereas a nonlinear signal will result in a deviation of DVV scatter diagram from the bisector line as illustrated in Figure 1(b). Furthermore, The minimal target variance is a measure for the amount of noise which is present in the time series (the prevalence of the stochastic component). Therefore, the distance of the DVV scatter diagram to the y-axis can be considered as a measure for predictability of the signal. These features of the DVV method provide a very convenient tool for the qualitative analysis in machine learning since the deviation from bisector line in the DVV scatter diagram can be used to indicate the changes in the signal nature before and after processing.

\section{SIMULATION RESULTS}

In this section, we set out to assess the qualitative performance, that is, a possible change in the signal nature in individual IMF after EMD method. For this purpose, we introduce a metric to measure the degree of such similarity, e.g., $\varepsilon=\sqrt{\left\langle\left(\sigma_{\text {ori }}^{* 2}\left(r_{\mathrm{d}}\right)-\sigma_{I M F}^{* 2}\left(r_{\mathrm{d}}\right)\right)^{2}\right\rangle_{\text {valid } r_{\mathrm{d}}}}$, where $\sigma_{\text {ori }}^{* 2}\left(r_{\mathrm{d}}\right)$ denotes the target variance at span $r_{\mathrm{d}}$ for the original signal whereas $\sigma_{I M F}^{* 2}\left(r_{\mathrm{d}}\right)$ denotes that for individual IMF. The smaller $\varepsilon$, namely, the better nature preservation that IMF has.

We perform such analysis on both the linear (2) and nonlinear (3) benchmark signals. For robustness, the analysis is obtained by averaging 100 independent trials.

Figure 2 illustrates the IMFs obtained from the AR(4) signal (3) after the decomposition. From the Figure, the top panel denotes the original signal (U) and there are five IMFs (C1-C5)in all, denoted as the second panel from the top to the second panel from the bottom. The bottom panel is the final residue $(\mathrm{R})$ in the sifting process, which can be ignored as it is a monotonic function or constant and serves no meaningful purpose. It can be observed that only the first IMF (C1) resembles the original signal (U), while others are not.

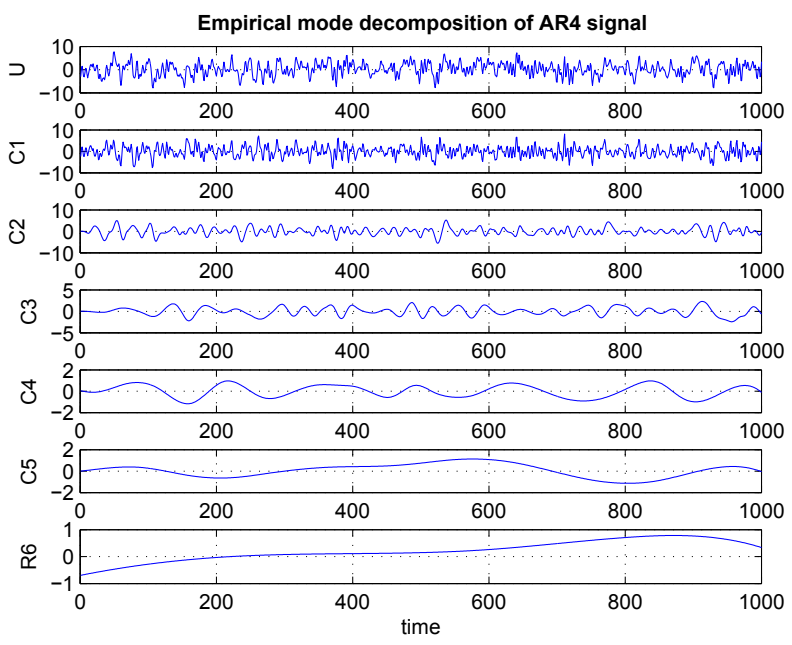

Fig. 2. The IMFs extracted from AR4 signal (2).

It is natural to wonder whether such resemblance only exists in time domain or not. To this cause, we perform the DVV analysis on individual IMF and the results are illustrated ${ }^{3}$ in Figure 3. It can be qualitatively shown from the Figure, the DVV scatter diagram for $1^{\text {st }}$ IMF is most similar to that for the original signal, which is illustrated in Figure 1(a). The DVV scatter diagrams for the other IMFs showed an increase in the predictability, illustrated by the fact that the curve in the diagram were closer to the y-axis. Quantitatively, the degree of similarity, $\varepsilon$, is also the smallest for $1^{\text {st }}$ IMF among all.

Finally, we perform the similar analysis on the nonlinear benchmark signal. Figure 4 illustrates the IMFs obtained from a nonlinear signal (3) after EMD method.

From the Figure, similar to the linear case, the first IMF (C1) has a greatest resemblance to the original signal (U). Figure 5 illustrates the DVV scatter diagrams for the first four IMFs. From the Figure, the $1^{\text {st }}$ IMF showed the highest degree of similarity to the original signal both qualitatively (curve resemblance) and quantitatively $(\varepsilon)$.

\section{CONCLUSIONS}

We have provided a quality assessment for the individual IMF of the empirical mode decomposition. By means of the 'delay vector variance' method, it has been shown that the first IMF not only resembles the original signal in the time domain, but also its DVV scatter diagram has the greatest degree of similarity to that of the original signal in the phase space in terms of curve resemblance and the quantitative metric, which measures the Euclidean distance of their phase space features (tar-

\footnotetext{
${ }^{3}$ For illustration purpose, we did not plot the DVV scatter diagrams for the last IMF and the residue.
} 

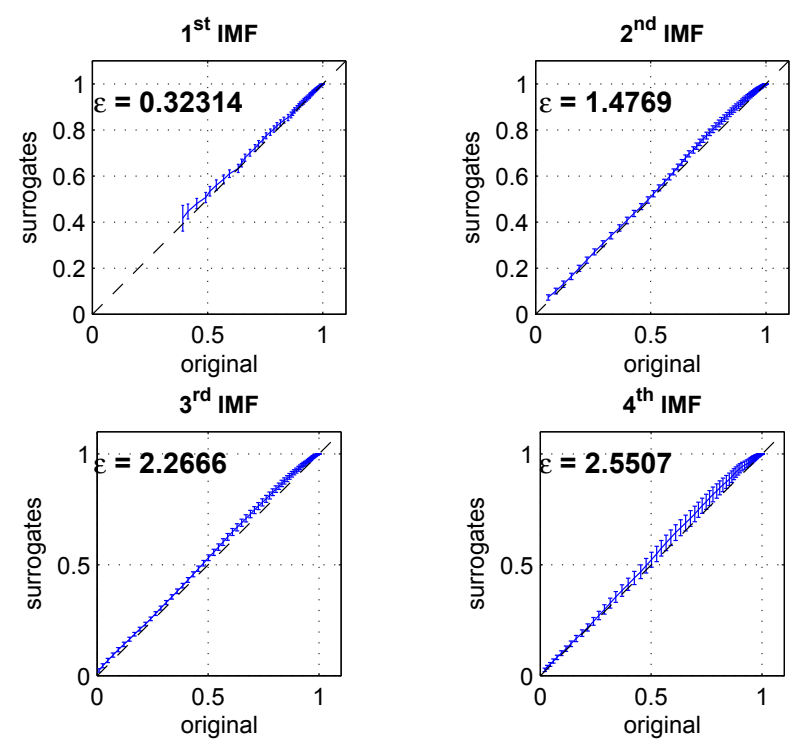

Fig. 3. The DVV scatter diagram for (clockwise from top left): $1^{\text {st }}$ IMF, $2^{\text {nd }}$ IMF, $4^{\text {th }}$ IMF and $3^{\text {rd }}$ IMF, from decomposition of AR(4) signal (2).

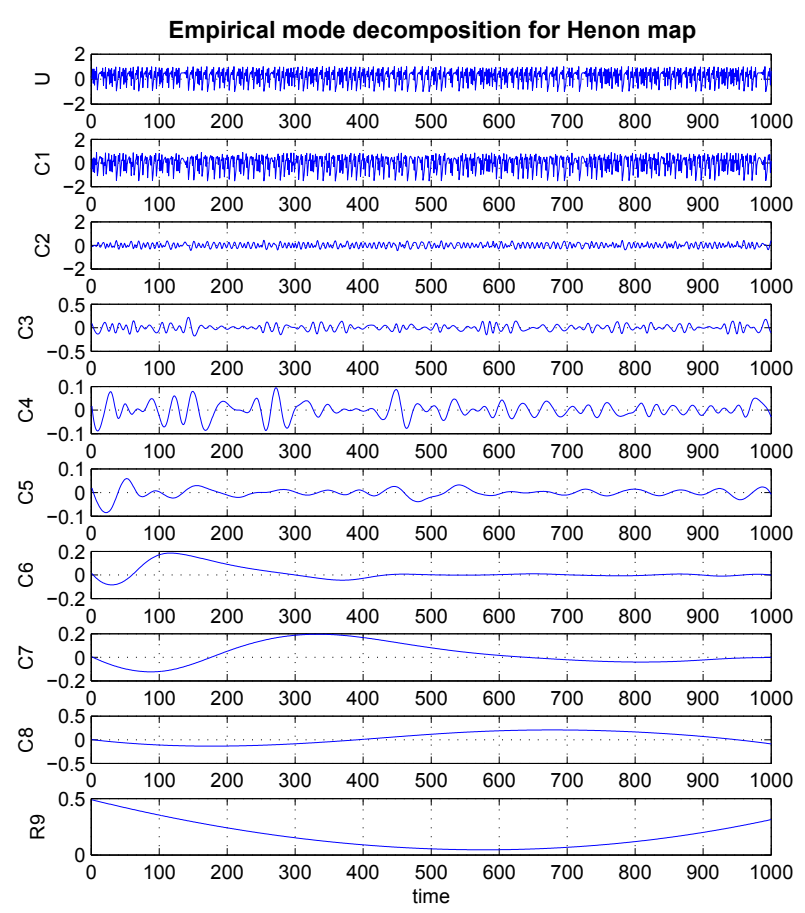

Fig. 4. The IMFs extracted from the Henon map signal (3).

get variance). The result will caution against the signal processing applications, denoising, that might consider removing or decreasing the importance of the first IMF when using EMD method.
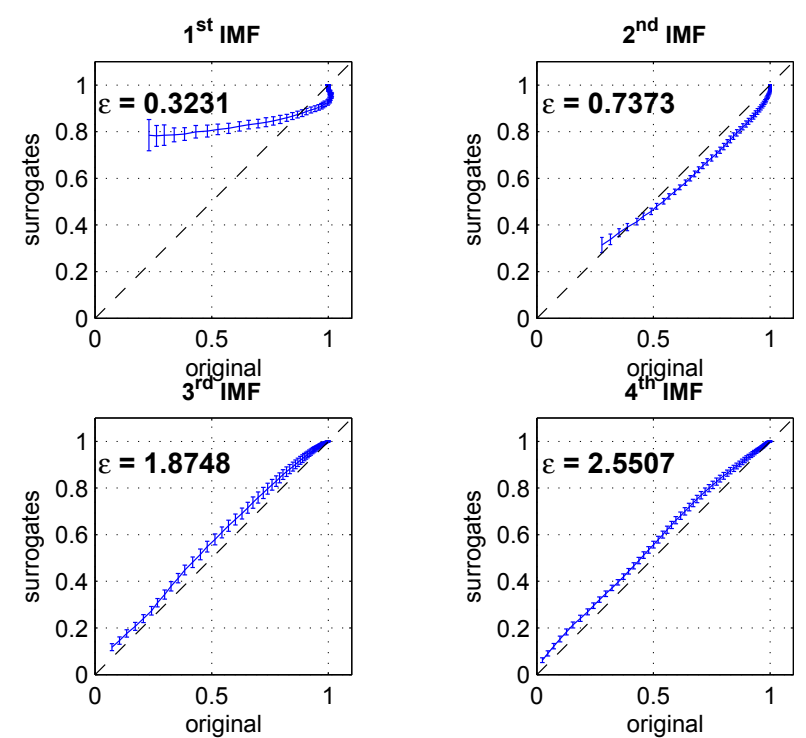

Fig. 5. The DVV scatter diagram for (clockwise from top left): $1^{\text {st }}$ IMF, $2^{\text {nd }}$ IMF, $4^{\text {th }}$ IMF and $3^{\text {rd }}$ IMF, from decomposition of henon map signal (3).

\section{REFERENCES}

[1] L. Stankovic and V. N. Ivanovic, "Further results on the minimum variance time-frequency distribution kernels," IEEE Transactions on Signal Processing, vol. 45, no. 6, pp. 16501656, 1997.

[2] L. Stankovic, "Time-frequency distributions with complex argument," IEEE Transactions on Signal Processing, vol. 50, no. 3, pp. 475-486, 2002.

[3] N. E. Huang, Z Shen, S. R. Long, M. C. Wu, H. H. Shin, Q. Zheng, N. Yen, C. C. Tung, and H. H. Liu, "The empirical mode decomposition and the hilbert spectrum for nonlinear and non-stationary time series analysis," Proc. R. Soc. Lond. A., 1998.

[4] T. Schreiber, "Interdisciplinary application of nonlinear time series methods," Phys. Rep., vol. 308, no. 1, pp. 1-64, 1999.

[5] T. Gautama, D.P. Mandic, and M.M. Van Hulle, "The delay vector variance method for detecting determinism and nonlinearity in time series," Physica D, vol. 190, no. 3-4, pp. 167-176, 2004.

[6] N. E. Huang and S.S.P. Shen, "Introduction to the hilbert-huang transform and its related mathematical problems, hilbert-huang transform and its application," Interdisciplinary Mathematical Science, World Scientific, , no. 1, 2005.

[7] L. Cao, "Practical method for determining the minimum embedding dimension of a scalar time series," Physica $D$, vol. 110, pp. 43-50, 1997.

[8] D.P. Mandic and J.A. Chambers, Recurrent Neural Networks for Prediction: Learning Algorithms Architecture and Stability, Wiley: Chichester, 2001.

[9] M. Henon, "A two-dimensional mapping with a strange attractor," Commun. Math. Phys., vol. 50, pp. 69-77, 1976. 